\title{
Flexography Printing for Organic Thin Film Transistors
}

\author{
Thomas Cosnahan a, Andrew A.R. Watt a \& Hazel E. Assender ${ }^{a} 1$ \\ a Department of Materials, University of Oxford, 16 Parks Road, Oxford, OX13PH, UK
}

\begin{abstract}
High throughput manufacture is of key importance for flexible electronics based on functional organic thin films. One challenge in particular is patterning at high speeds. In this paper we present results of organic transistors created using in-vacuum flexography patterning. This combines an industry standard patterning method with resolution good enough to create functional devices.

Optimisation results of the patterning process and analysis of the printed devices will be presented. Specifically the impression force and printing speeds are varied to optimise the print quality, which is quantified using image analysis. These results inform the theory of ink transfer and the limits of this technique in terms of resolution and process parameters. As proof of concept, common gate, top contact transistors are created as per previous group results (1) but without the use of shadow masks to create the aluminium contacts. Masked and metal patterned devices are compared and show the compatibility of this technique for our transistors; while increasing the production throughput the performance is not diminished. This paper will also demonstrate that range of applications this production technique could have.
\end{abstract}

1. Taylor DM, Patchett ER, Williams A, Ding Z, Assender HE, Morrison JJ, et al. Fabrication and simulation of organic transistors and functional circuits. Chemical Physics. $2015 \mathrm{Jul}$ 29;456:85-92.

Keywords: Flexography, diacrylate, organic thin film transistors, vacuum, patterning

\section{Introduction}

This research paper is focused on the question of feasibility for commercial application of flexography for deposition of patterned layers for flexible electronics. It investigates the compatibility of the materials with the organic electronics, and presents arguments for the viability of the production method for organic electronics (OE), in particular for organic thin film transistors (OTFT's). By bringing together organic electronics with a mass production method, the results expand scientific understanding of the material compatibility and process mechanism viability. Viability and compatibility depend on the particular device requirements and process aims, so they are examined in the following sections for the particular case of an organic dielectric layer, which is a diacrylate: tripropylene glycol diacrylate (TPGDA), for OTFT's with a bottom gate top contact planar structure.

\footnotetext{
* Corresponding author. Tel.: +0-000-000-0000 ; fax: +0-000-000-0000
}

E-mail address: hazel.assender@materials.ox.ac.uk 


\subsection{Viability of Flexography}

Viability experiments test the production process' abilities in order to determine whether the process could be a method of production. If a process is viable its mechanisms and physical properties will be understood sufficiently such that the required $\mathrm{OE}$ device can be fabricated. An unviable production process could not create the required device due to the physical limitations of the process. Investigating viability requires an understanding of the origins of the process, the level of industrial know-how and skill required, and the physical mechanistic constraints. This section expands on these themes for flexography printing.

Flexography dates to the printing press industry at the turn of the $20^{\text {th }}$ century and has since been developed and improved so that it now holds a large market share of the graphics industry. $(1,2)$ Its success in the graphics printing field lay in the versatility of ink viscosities that could be used and the anilox system which readily controlled the amount of ink transferred from inking system to plate and subsequently to the substrate, see figure 1 . The deposited ink thickness is about $1 \mu \mathrm{m}$, according to a key textbook of graphics printing (3), which is similar or slightly thicker than the thickness required for polymer dielectric layers. (4) The lateral printed dimensions that are achievable using standard graphic flexography (in the order of a few 10's microns) are similarly applicable with large area electronics, but may be limiting for devices which require higher resolution patterning. It is evident from this industrial know-how that flexography could be successfully applied to some OE thin film layers.

These features make flexography a viable process for OE production. As a mature production process, the wealth of industrial know-how creates a fertile environment for trials to be conducted. Furthermore the non-aesthetic but much finer tolerances required by OE layers have led to research to make this printing press a suitable deposition system. Research (e.g. (5)) has clarified the process parameters required for reliable, accurate and reproducible printing, in particular on commercialscale trials. (6)(7) As the flexography process is further specified by commercial scale trials and process variable effects verified, it has become apparent that there are still questions surrounding the exact nature of the ink transfer mechanism between patterned rollers, and conflicting results exist over the effect of the process parameters such as velocity and impression force.(8-10) Advancing the scientifically testable understanding of the ink transfer mechanism could lead to improvements in the achievable resolution (for instance by controlling edge dewetting $(11,12)$ ) and so investigating flexography's viability could increase the feasibility of flexography patterning for organic electronics. In this work flexography is performed in vacuum, such as would be used for deposition with solvent free processes, so the effects of entrained air within the printing nip is removed, leaving only the printing plate and ink rheology to affect the ink transfer.(5) 




Fig. 1 Schematic of the principal components of flexography printing displaying the movement of ink from a micro-engraved anilox to the printing plate, where the ink is held on the raised relief pattern and then transferred to the substrate which is held on an impression drum. This schematic shows a complete ink transfer from the plate to the substrate, which is unrealistic, and also omits the anilox inking process. See $(3,13)$ for further descriptions.

\subsection{Compatibility of Di-Acrylate Dielectric}

Compatibility experiments test the effect of the production process on the materials involved to determine whether the process could create the required device properties. A compatible process would create $\mathrm{OE}$ devices with material characteristics as similar to the material selection ideal as possible. An incompatible production process cannot fabricate the $\mathrm{OE}$ device with materials characteristics good enough to function. For this industrial scale printing technique to be feasible for OE production, the deposited thin film materials must be compatible not only with the OE but also with the flexography tools and process. In this section the compatibility of TPGDA, which has been shown to lead to good OTFT performance, with flexography is introduced by assessing the reported scientific research on the materials in question.

In this investigation a silicone (polydimethylsiloxane (PDMS)) print plate was used in place of the photo-curable rubber plate typically used industrially. PDMS was chosen for its ease of fabrication and pattern-ability. $(14,15)$ The printed material should not swell or react with the printing plate. PDMS is chemically inert but will swell in nonpolar liquids. The compatibility of the chemicals printed with the printing plate needs to be tested, as swelling could lead to pattern distortion.

Previous work has shown the material characteristics of TPGDA can create smooth organic dielectrics when deposited using flash evaporation, and although it does not have high relative permittivity, when used as a gate dielectric layer in OTFT's the impressive bulk properties lead to very low hysteresis devices with good mobility when combined with a nonpolar buffer layer.(16) The direct deposition of the monomer within a vacuum environment, followed by curing of the cryocondensed liquid allows a pin-hole free smooth dielectric layer to be formed over large areas. Using the liquid monomer as the ink in the flexography process could produce similarly compatible thin films, but now with patterning, to allow exposed areas for electric connections (vias) between devices. The ink transfer mechanism should not adversely affect the monomer if shear forces are kept low enough to avoid degradation. Furthermore the printing pressure of the ink impression should not damage the existing device layer structure. 
The existing coating method by flash evaporation relies on curing the monomer by the formation of free radicals e.g. by UV radiation, e-beam or plasma(16). This post-printing cure following flexography printing will allow pattern deposition of di-acrylate dielectrics. As a consequence, no drying or solvent removal steps will be required due to the direct deposition, which increases the throughput which can be achieved, and minimizes the risk of pin-hole formation on solvent removal. Other flexographic inks show a relationship between the process parameters and material properties (17), this compatibility remains to be investigated for these dielectric materials.

\subsection{Mass Manufacturing of Organic Electronics}

OTFT's produced using flexography will be feasible if the flexography deposition process can be shown to be compatible and viable. As a union of organic electronics and mass production a patchwork of literature has been reviewed to show that there is a theoretical basis for conducting experimental trials to deduce whether the process could be feasible. Furthermore, the results may lead to further understanding of the mechanism of ink transfer within flexography which could subsequently increase the viability and feasibility of this promising production process.

In this paper we will show the results of using this technique within a vacuum to create patterned functional dielectric layers for OTFT's. In the next section the fabrication and analysis process is described in more detail to show that flexography printing in vacuum can be used to create thin films which are compatible with organic devices. The thin film properties are compared with thin film coatings, and then these results are discussed to conclude whether flexography is a viable and compatible production process.

\section{Methods}

A simple laboratory scale flexography rig was created, see figure 2 , which fitted into a laboratoryscale vacuum chamber. In a single rotation of the impression drum the flexography plate, or print head, rotated counter-wise to be impressed upon the substrate fixed to the process drum. In a single rotation, first the print plate was inked by an inked membrane, of length equal to the plate circumference, attached to the substrate (which mimics the anilox roller). Then the plate transferred this liquid monomer onto the PET substrate or silicon chip in the pattern defined by the PDMS print head. This liquid thin film on the process drum passed an Argon plasma which cured the monomer to create the patterned acrylate dielectric polymer thin film. 


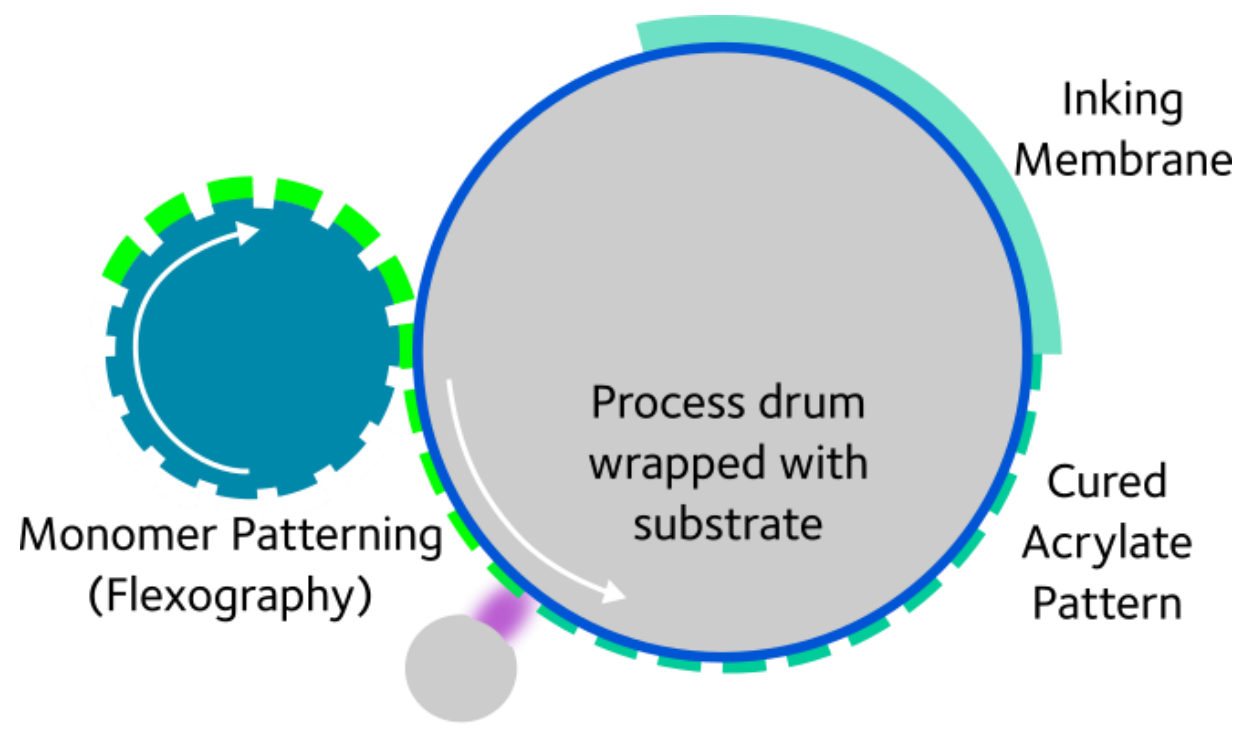

Plasma Treatment

Fig. 2 Schematic of the experimental lab flexography set up with plasma curing following the patterning of monomer liquid to create the cured acrylate pattern. This was all performed in a medium vacuum (0.1-1Pa)

The dielectric monomer, TPGDA, was purchased from Sigma Aldrich and used as-received. The parallel plate thin film capacitor had a common bottom aluminium electrode which was a strip of thermally evaporated aluminium on a polyethylene naphtalate (PEN) substrate. Regular stripes of diacrylate were patterned in the cross direction across the common bottom electrode. The top electrode was also aluminium which was thermally evaporated onto the patterned dielectric layer, through a shadow mask and defined the area of capacitance measurement. Capacitance of the patterned TPGDA was measured with capacitance meter (Tenma 72-7750). Thickness and line roughness measurements were taken on a Dektak ${ }^{\circledR}$ stylus profilometer with the patterned acrylate on polished silicon wafer.

The solubility parameter of TPGDA and PDMS were estimated using the Fedors additive function method, see Table 1.(18) An immersion swelling test over 24 hours was also conducted to corroborate the theoretical estimation, as per Lee et. al's work on PDMS swelling.(19)

Table 1 Fedors additive function summation table for TPGDA monomer group contributions.

\begin{tabular}{llll}
\hline Group & Number & $\Delta$ ei cal/mol & $\Delta$ vi cm3/mol \\
\hline CH & 5 & 820 & -1 \\
CH2 & 2 & 1180 & 16.1 \\
CH3 & 3 & 1125 & 33.5 \\
CO & 2 & 4150 & 10.8 \\
CO2 & 2 & 4300 & 18 \\
H2C= & 2 & 1030 & 28.5 \\
$\mathbf{1 6}$ skeletal atoms & 16 & & 2 \\
Total & & 28795 & 274.3 \\
\hline
\end{tabular}

\section{Results}

The diacrylate structure is known and TPGDA's solubility parameter was found to $10.25 \mathrm{cal}^{1 / 2} \cdot \mathrm{cm}^{3 / 2}$, which is significantly different from that of PDMS: $7.3 \mathrm{cal}^{1 / 2} \cdot \mathrm{cm}^{3 / 2}$.(19) This suggests that swelling of the printing plate by the ink should be negligible. Fedors claims a $10 \%$ accuracy when compared with experimental values, assuming $25^{\circ} \mathrm{C}$ and that the vaporisation energy of the glass and liquid state are 
the same.(18) This theoretical finding has been corroborated by experimental analysis:over 24 hours there was no swelling observed in a PDMS sample.

The viscosity of the diacrylate used was $0.01 \mathrm{mPa}$.S (sales literature). This is at the lower end of the standard viscosity window for flexography (0.05-05Pa.s)(3) but the printed patterns still have good fidelity to the patterned plate, see figure 3 . Thickness and roughness measurement results are shown in Table 2, with the maximum peaks being five times larger than the depth of the minimum valleys. The capacitance was measured to be between 11 and $13 \mathrm{nF} . \mathrm{cm}^{-2}$ over three patterned diacrylate stripes.

Table 2 Average thickness and line roughness measurements of the patterned diacrylate made using Dektak stylus profilometry and the standard deviation of the five measurements.

\begin{tabular}{llll}
\hline Measurement & Average & Standard Deviation & unit \\
\hline Thickness & 40.67 & 0.70 & $\mathrm{~nm}$ \\
RMS Roughness & 5.88 & 8.43 & $\mathrm{~nm}$ \\
Peak to Peak Height & 36.14 & 6.04 & $\mathrm{~nm}$ \\
\hline
\end{tabular}

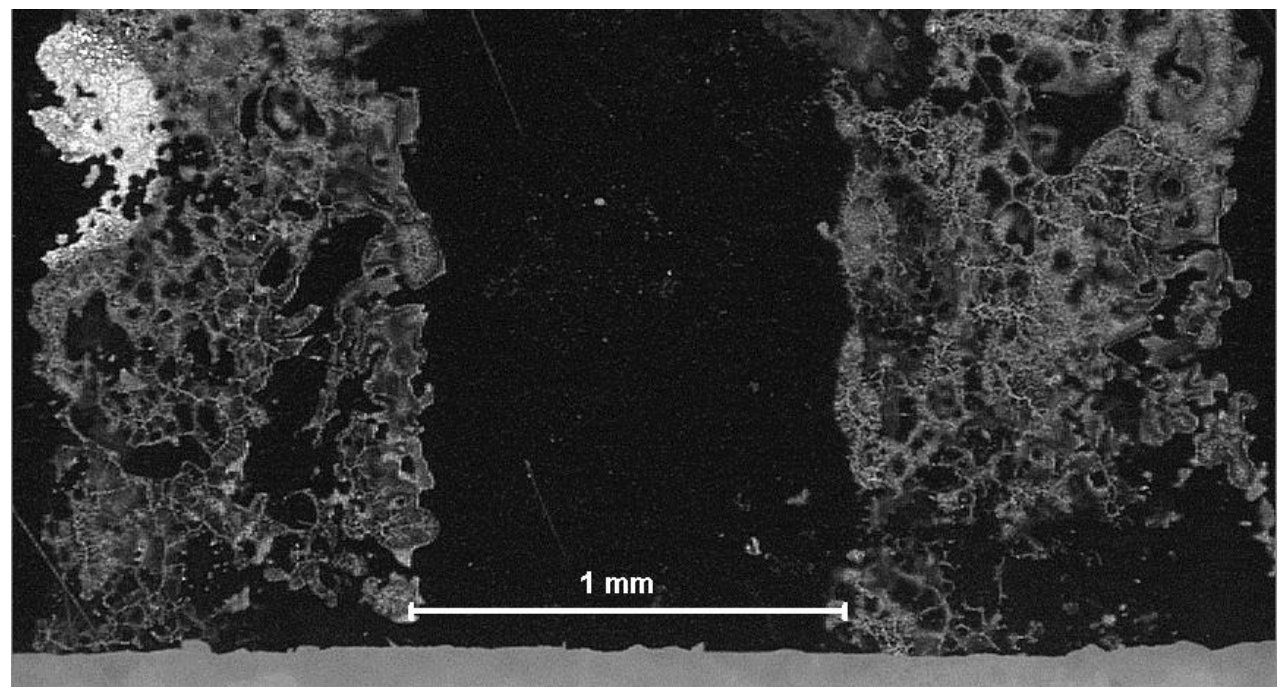

Fig. 3 Optical micrograph of the patterned diacrylate on a Si chip (darker regions) $1 \mathrm{~mm}$ stripe patterned diacrylate has good pattern fidelity but clear surface roughness.

\section{Discussion}

The Hildebrand parameter found is above the Lee et. al.'s definition of low solubility limit of $9.9 \mathrm{cal}^{1 / 2} \mathrm{~cm}^{-3 / 2}$ (19) and is similar to polymers which have a near zero swelling ratio with PDMS. Empirical swelling test corroboration also means that the printing ink, that is the diacrylate monomer, will not distort the print plate during a print, adding to the compatibility of the method. The Hansen solubility factor could inform more on the type of swelling possible, not all solvents of similar Hildebrand solubility parameter lead to swelling with PDMS, as this parameter is too broad a brush to paint an accurate correlation. However enough is known from Hansen solubility parameter analysis that PDMS swells in the presence of non-polar chemistry, which the monomer in question is not.Using a common material like PDMS meant the solvent compatibility was more readily calculable, and resourcing and fabricating the printing plates simpler. These add to the compatibility of the process with in a lab environment but would need to be adapted for industry where photopolymer rubbers are currently used as standard.

The low viscosity of the monomer could mean that similar print result would be difficult to achieve in industry with a standard anilox inking system. Using an inking membrane, instead of a standard gravure inking method, removed the extent of stringency on the viscosity range, as the print pressure will have a larger impact on the 
ink transfer from the membrane than with an anilox roll, where ink 'lift out' of the cells is strongly affected by the ink viscosity.(5)

The thickness and roughness measurements shed light on the printing and curing steps, and help determine the viability of the process. Typically graphic inks have a thickness of approximately $1 \mu \mathrm{m}$ but in this print, the diacrylate thickness was only $40.67 \pm 0.7 \mathrm{~nm}$. Thinner dielectric layers, provided leakage current can be avoided, are desirable for OTFTs, as this will reduce the required gate voltage. With a more viscous monomer liquid a thicker deposition could be observed.

Tests with a longer levelling time and higher print pressures before curing of the patterned monomer on the substrate resulted in similar thickness and roughness measurements. This suggests, then, that it is the plasma curing step, rather than the ink transfer process, that had a large influence on the surface topography, and under certain curing conditions such surface roughness has been observed previously.(20) It is possible the top electrode formed a much larger contact area upon the diacrylate roughness. This anomaly could lead to the surprisingly high capacitance values (of the same order of magnitude to coated flash evaporated values), despite the layer thickness being an order of magnitude less. That said, the roughness was skewed, with shallow valleys separated by sharp peaks, so that the valleys would not cause pinhole defects. However the topography of the high capacitance diacrylate would be too rough to achieve the highly ordered crystalline organic semiconductor at the interface that leads to high mobility OTFT's.

Although there are currently some compatibility problems with the curing process which would need to be improved for this monomer to be used as a high quality dielectric layer in OTFT's, the results imply that continuous and thin polymer films with high capacitance can be created with pattern printing process.

\section{Conclusion}

The results of this viability and compatibility investigation have shown that flexography is a viable production method for creating organic dielectric patterned thin films. However these initial results have highlighted that the cured diacrylate material with this first set of conditions leads to a surface that is too rough to be compatible with OTFT's. Further optimisation of the plasma curing step could improve the surface roughness to match the good pattern fidelity despite the low viscosity of the monomer. The surprisingly high capacitance values, given how thin the patterned diacrylate was, are attributed to the surface roughness increasing the electrode area but it could also be a curing effect or due to limitations in the simple capacitance measurement experiment.

This research paper has also shown that pursuing compatibility and viability can lead to increasing the throughput or reducing cost in some circumstances, as shown with the removal of the drying step with this in-vacuum direct deposition process. Furthermore, investigating the viability of flexography has demonstrated a process that is not limited to OTFT's, and other OE device layers could be fabricated in this way.

\section{Acknowledgements}

The authors would like to thank the Engineering and Physical Sciences Research Council for financial support.

\section{References}

1. Benchmarking and Worldwide Market Trends for Flexographic Printing [Internet]. [cited 2017 Aug 1]. Available from: http://www.npes.org/primirresearch/researchstudies/content.aspx?Topic=Benchmarking_and_Worl dwide_Market_Trends_for_Flexographic_Printing

2. Flexographic Printing Worldwide Market Data and Packaging Industry Trends [Internet]. Package Printing. [cited 2017 Aug 1]. Available from: http://www.packageprinting.com/article/benchmarking-worldwide-market-trends-flexographicprinting-25008604/ 
3. Kipphan $\mathrm{H}$, editor. Handbook of print media: technologies and production methods [Internet]. Berlin ; New York: Springer; 2001. 1207 p. Available from: doi:10.1007/978-3-540-29900-4

4. Abbas G a., Ding Z, Assender HE, Morrison JJ, Yeates SG, Patchett ER, et al. A high-yielding evaporation-based process for organic transistors based on the semiconductor DNTT. Org Electron. 2014 Sep;15(9):1998-2006.

5. Bould DC, Hamblyn SM, Gethin DT, Claypole TC. Effect of impression pressure and anilox specification on solid and halftone density. Proc Inst Mech Eng Part B J Eng Manuf. 2011 May 1;225(5):699-709.

6. Bould DC, Claypole TC, Bohan MFJ. An investigation into plate deformation in flexographic printing. Proc Inst Mech Eng Part B J Eng Manuf. 2004 Nov 1;218(11):1499-511.

7. Deganello D, Cherry JA, Gethin DT, Claypole TC. Patterning of micro-scale conductive networks using reel-to-reel flexographic printing. Thin Solid Films. 2010 Aug 31;518(21):6113-6.

8. De Grâce JH, Mangin PJ, International Association of Research Institutes for the Graphic Arts Industry, Research Conference. A mechanistic approach to ink transfer. Part II. Pointe Claire, Québec: Pulp and Paper Research Institute of Canada; 1987.

9. Dodds S, Carvalho MS, Kumar S. The dynamics of three-dimensional liquid bridges with pinned and moving contact lines. J Fluid Mech. 2012 Sep;707:521-40.

10. Kumar S. Liquid Transfer in Printing Processes: Liquid Bridges with Moving Contact Lines. Annu Rev Fluid Mech. 2015;47(1):67-94.

11. Cheng W, Park N, Walter MT, Hartman MR, Luo D. Nanopatterning self-assembled nanoparticle superlattices by moulding microdroplets. Nat Nanotechnol. 2008 Nov;3(11):682-90.

12. Kwak MK, Shin KH, Yoon EY, Suh KY. Fabrication of conductive metal lines by plate-to-roll pattern transfer utilizing edge dewetting and flexographic printing. J Colloid Interface Sci. 2010 Mar 1;343(1):301-5.

13. Xia Y, Whitesides GM. Soft Lithography. Annu Rev Mater Sci. 1998;28(1):153-84.

14. Nodin MN, Yusof MS. A Preliminary Study of PDMS Stamp towards Flexography Printing Technique: An Overview. Adv Mater Res. 2013 Nov;844:201-4.

15. Abbas G, Ding Z, Mallik K, Assender H, Taylor DM. Hysteresis-Free Vacuum-Processed Acrylate Pentacene Thin-Film Transistors. IEEE Electron Device Lett. 2013 Feb;34(2):268-270.

16. Joyce MJ, lii DPDF, Avuthu SGR, Emamian S, Eshkeiti A, Atashbar DM, et al. Contribution of Flexo Process Variables to Fine Line Ag Electrode Performance. In IJERT; 2014 [cited 2017 Feb 6]. Available from: http://www.ijert.org/view-pdf/11151/contribution-of-flexo-process-variables-tofine-line-ag-electrode-performance

17. Fedors RF. A method for estimating both the solubility parameters and molar volumes of liquids. Polym Eng Sci. 1974 Feb 1;14(2):147-54.

18. Lee JN, Park C, Whitesides GM. Solvent Compatibility of Poly(dimethylsiloxane)-Based Microfluidic Devices. Anal Chem. 2003 Dec;75(23):6544-54. 
19. Affinito JD, Eufinger S, Gross ME, Graff GL, Martin PM. PML/oxide/PML barrier layer performance differences arising from use of UV or electron beam polymerization of the PML layers. Thin Solid Films. 1997 Oct 31;308-309:19-25. 